\title{
Bibliothèques captives. Politiques du livre dans les langues de l'État espagnol
}

Captive libraries: book policies in the languages of the Spanish state

\section{María Fátima Rodriguez}

\section{(2) OpenEdition}

\section{Journals}

Édition électronique

URL : http://journals.openedition.org//bl/420

DOI : $10.4000 / \mathrm{lbl} .420$

ISSN : 2727-9383

Éditeur

Université de Bretagne Occidentale - UBO

\section{Édition imprimée}

Date de publication : 1 mars 2016

Pagination : 15-28

ISBN : 979-10-92331-24-0

ISSN : $1270-2412$

Référence électronique

María Fátima Rodriguez, "Bibliothèques captives. Politiques du livre dans les langues de l'État espagnol », La Bretagne Linguistique [En ligne], 20 | 2016, mis en ligne le 01 mai 2020, consulté le 01 octobre 2020. URL : http://journals.openedition.org/lbl/420 ; DOI : https://doi.org/10.4000//bl.420

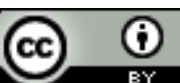

La Bretagne Linguistique est mise à disposition selon les termes de la Licence Creative Commons Attribution 4.0 International. 


\section{Bibliothèques captives. Politiques du livre dans les langues de l'État espagnol}

\section{$\mathrm{P}$}

our ordonner les interrogations que le titre même de ce travail peut susciter, je commencerai par l'anecdote et tenterai d'avancer dans la direction du principe et de la remarque.

Il y a six ans, une information romanesque inonde littéralement les médias en Galice. Romanesque, elle l'aurait été, si elle n'avait pas touché de près ou de loin le portefeuille du contribuable, à un moment où pointait la crise économique que l'Espagne connaît encore.

Le fait a lieu dans les cryptes du Centre de recherches Ramón Piñeiro, du nom d'un intellectuel galicien (1915-1990), artisan du «nationalisme culturel ». Cet institut, fondé en 1993, est implanté à Saint Jacques de Compostelle et financé par la Xunta de Galicia (à travers la Secretaría Xeral de Política Lingüística). Le lieu renfermait deux millions de livres de sa production, non-commercialisés, fruits - défendus - de programmes et bourses de recherche, de registres patrimoniaux à facsimiler ou de l'exhumation d'un certain nombre d'écrivains significatifs de la Galice.

Dans la mesure où la région compte un million et demi de lecteurs, en cas de répartition symbolique, nous aurions droit, au moins, à un livre par tête, défiant toutes les campagnes de promotion de la lecture menées par l'UNESCO, même les plus ambitieuses ${ }^{1}$. Il ne faut pas omettre un détail essentiel: ces exemplaires étaient tous rédigés en galicien.

* Professeur d'espagnol, HCTI, UBO.

1. Nous renvoyons aux études de Robert EsCARPIT et Ronald BARKER sur les campagnes pour la lecture, matérialisées dans La faim de lire, Paris, Presses Universitaires de France, 1973. 
On peut nous objecter que l'accès potentiel à ces ouvrages est sans doute supérieur à celui d'un grand nombre de volumes du biblio-bunker de Tolbiac, à Paris, sans compter bien d'autres de la Biblioteca Nacional de España où, à l'aube du XXI ${ }^{\mathrm{e}}$ siècle, il existait encore des archives scellées (l'archive de la franc-maçonnerie, par exemple). Car, accessibles, ils le sont: il suffit de se présenter dans le siège institutionnel, aux abords de Compostelle jusqu'à cette année, pour récupérer un ou plusieurs de ces documents en état de captivité, à condition qu'ils ne soient pas épuisés (condition irréalisable, dans la mesure où ils ne voient pas la lumière), ou qu'un prix de vente au public leur soit assigné, chose tout aussi exceptionnelle, puisqu'ils ne sont pas mis sur le marché.

Il existe un projet de Bibliothèque nationale de Galice qui suppose, non sans un certain optimisme, que les mêmes producteurs de ces livres aujourd'hui en disgrâce, par le biais d'une acrobatie financière d'auto-achat, investiraient presque cinq millions d'euros en rayonnage bibliographique de la Cidade da Cultura (Cité de la Culture), surnommée Monstre de Gaias (du nom du lieu où elle fut érigée), un puits sans fond qui a coûté au jour d'aujourd'hui 300 millions d'euros et dont les travaux inachevés ont été interrompus par décret en mars $2013^{2}$. Le célèbre mastodonte culturel fut conçu par Peter Einsenman et, à l'époque dont on parle, il avait déjà coûté quarante-cinq millions d'euros; c'est le prix approximatif, par ailleurs, de ces deux millions et demi de livres, dans une éventuelle opération de vente, plus du double du budget alloué par cette même Communauté autonome à sa politique linguistique dans son exercice annuel ${ }^{3}$ mais ces vicissitudes livresques ne s'achèvent pas ici.

Car une option inouïe apparaît ces derniers jours: c'est un autre fonds bibliographique qui rayonnera, pardonnez-moi le jeu de mots facile, dans les rayonnages de Gaias: celui des Caisses d'épargne de la région; malmenées par la crise, elles se sont désolidarisées de leur activité socioculturelle et ont confié au gouvernement régional un trésor de 3500 pièces de joyaux bibliographiques allant du XVe siècle à nos jours. Le sort des volumes confinés reste ainsi plus qu'incertain.

Ce genre de cas ne permet sans doute pas de se faire une idée juste de l'évolution des actions politiques destinées à la préservation d'une langue vernaculaire en Espagne. De l'extérieur, on pourrait même considérer

2. Ibid. «A Xunta busca unha saída...», http://www.culturagalega.org/noticia. php?id=7307. Consulté le 13février 2013.

3. Au moment de présenter cette communication, le budget voté est de 23247547 euros. Il est consultable sur le site de la Consellería de Economía e Facenda de la Xunta de Galicia (www.economiaefacenda.org). 
ces exemples dépourvus du moindre intérêt académique. Mais l'anecdote est pertinente dès lors qu'elle montre les contradictions d'un système, concrétisé ici dans la convergence de deux attitudes opposées: d'une part les pratiques des pouvoirs publics et intellectuels, animés par une volonté de doter nos langues d'une production scientifique écrite conséquente, et d'autre part, l'escamotage, la faible ou nulle visibilité de cette production. Les deux répondent à mon sens à une perception pendulaire de la langue, oscillant entre l'engagement théorique externe pour leur conservation et une réaction interne invalidante. Deux forces opposées en cohabitation, entre la devanture et la mésaventure, entre le compte et le mécompte.

En prenant pied sur ces exemples, pour le moins curieux, je voudrais essayer de rattacher quelques maillons de la chaîne évolutive des langues de l'État espagnol dans la sphère publique, depuis leur légitimation en 1978 par voie constitutionnelle. Le but de cette communication est de poser des problèmes liés aux échanges et aux constructions sociales, partant de la situation de la péninsule ibérique et considérant la capacité d'exportation de ces initiatives de régulation de la cohabitation linguistique, notamment en Amérique latine ${ }^{4}$. Il est en effet indéniable, à trente-cinq ans de distance, que notre modèle territorial à base linguistique inspire d'autres initiatives à plusieurs échelles, supranationale et transnationale, même si son impact dans la population espagnole est difficilement évaluable.

D'entrée, évoquer ce processus est faire appel à l'un des exemples de planification linguistique les plus originaux ${ }^{5}$ et les plus controversés à la fois, du moins du point de vue de l'opinion publique.

\section{Langues et État: les origines d'une légitimation}

Il y a déjà plus de trente ans que l'Espagne a vu naître les dispositifs institutionnels pour le développement des langues catalane, galicienne et euskera. Et d'une manière bien plus diffuse du bable (Asturies) et du

4. C'est le cas de la langue guarani. Les forums internationaux sur la politique linguistique tenus tous les ans à Asuncion depuis 2008 comptent des spécialistes galiciens et catalans. Ces informations émanent de deux entretiens personnels avec Ana Miranda, ancienne députée au Parlement européen, et de Susy Delgado, écrivaine et journaliste paraguayenne, ancienne chargée de mission pour le développement de la langue guarani.

5. Charlotte HofFMAnN parle de « fascinating exemple of language planning both in terms of policy formulation and in terms of policy implementation ", "Monolingualism and Bilingualism, Cultural Pluralism and National Identity: Twenty Years of Language Planning of Contemporary Spain», dans Sue Wright (dir.), Monolinguism and Bilinguism. Lessons from Canada and Spain, Clevedon-Philadelphia, Multilingual Matters, 1996, p. 59. 
valencien. De tels dispositifs s'accompagnaient de moyens financiers conséquents. Et efficaces, car en à peine six années (entre 1980 et 1986) seront dessinées les politiques linguistiques des trois communautés autonomes identifiées comme "historiques »: la Catalogne, la Galice et Euskadi.

Quand bien même les décisions préalables à la rédaction des textes constitutionnels ne dépendaient pas des facteurs linguistiques, il convient de rappeler un fait clé: sur les 17 communautés et les deux villes autonomes déterminées dans la nouvelle répartition politico-territoriale d'un État de droit flambant neuf après la mort du dictateur Francisco Franco, la moitié vivait en situation de bilinguisme ( $40 \%$ de la population espagnole et $25 \%$ de locuteurs bilingues), concentrés ou répartis, selon les lucarnes, sur un cinquième du territoire physique espagnol.

Le fait est que la diversité linguistique apparaît intégrée pour la première fois sous un ordonnancement juridique commun, que nous rappelons brièvement ici.

Sur les 158 chapitres de la Constitution, trois sont consacrés à la question des langues:

Article 3.

1. Le castillan est la langue espagnole officielle de l'État. Tous les Espagnols ont le devoir de la savoir et le droit de l'utiliser.

2. Les autres langues espagnoles seront également officielles dans les Communautés autonomes respectives, conformément à leurs statuts.

3. La richesse des différentes modalités linguistiques de l'Espagne est un patrimoine culturel qui doit être l'objet d'une protection et d'un respect particuliers.

Article 20.

On reconnait et on protège le droit:

3. La loi réglementera l'organisation et le contrôle parlementaire des moyens de communication sociale dépendant de l'État ou d'une entité publique et garantira l'accès à ces moyens aux groupes sociaux et politiques significatifs, dans le respect du pluralisme de la société et des différentes langues de l'Espagne.

4. Ces libertés trouvent leur limite dans le respect des droits reconnus au présent titre, dans les préceptes des lois qui le développent et, en particulier, dans le droit à l'honneur, à l'intimité, à sa propre image et à la protection de la jeunesse et de l'enfance. 
Article 147.

1. Selon les termes de la présente Constitution, les statuts seront la norme institutionnelle de base de chaque Communauté autonome et l'État les reconnaîtra et les protégera comme partie intégrante de son ordre juridique.

2. Les statuts d'autonomie devront contenir:

a) le nom de la Communauté qui correspondra le mieux à son identité historique.

Article 148.

Les Communautés autonomes pourront assumer des compétences dans les matières suivantes:

$15^{\mathrm{e}}$ les musées, les bibliothèques et les conservatoires de musique présentant un intérêt pour la Communauté autonome;

$16^{\mathrm{e}}$ le patrimoine monumental présentant un intérêt pour la Communauté autonome;

$17^{\mathrm{e}}$ le développement de la culture, de la recherche et, s'il y a lieu, de l'enseignement de la langue de la Communauté autonome;

$18^{\mathrm{e}}$ la promotion et l'aménagement du tourisme sur leur territoire;

$19^{\mathrm{e}}$ la promotion du sport et l'utilisation adéquate des loisirs ${ }^{6}$.

2. Les statuts d'autonomie devront contenir:

La dénomination de la Communauté qui corresponde le mieux à son identité historique.

L'article $148^{7}$, en fixant les compétences des CC. AA. préconise « le développement de la culture, de la recherche et, le cas échéant, l'enseignement de la langue de la Communauté Autonome ».

Cette initiative apparaît entre le Tourisme, la conservation du patrimoine monumental, la promotion du sport et la correcte utilisation des loisirs.

Si j'évoque ici ces textes racinaires, c'est qu'ils concrétisent plusieurs constructions mentales axées sur les concepts suivants :

1. Pluralisme

2. Identité

3. Officiel

4. Diversité

En adoptant ces concepts, le binôme identité-différence est démonté pour ouvrir la porte à une notion implicite, la multiculturalité, entendue

6. http://www.aelpa.org/documentos/legislacion_nacional/Constitucion_fra.pdf

7. Ibid. p. 46. 
comme un agrégat de cultures partageant le même territoire géographique et symbolique.

Première construction: prise en considération de la singularité d'un territoire linguistique au sein d'un territoire géographique et historique, identifiant de manière schématique un espace physique à un espace linguistique, ce qui diverge, par exemple, de la Charte Européenne des langues régionales et minoritaires ${ }^{8}$. Les droits légaux s'appliquent aux territoires, non pas aux locuteurs (la population immigrée peut sentir ses droits bafoués par les politiques des CC. AA., de la même manière qu'un locuteur de ces langues n'a aucun droit en dehors de sa circonscription linguistique).

Les premières contre-offensives à cette entreprise se déclenchent en 1981, puis en 1994, menée cette fois-ci par le directeur de la Real Academia Española d'alors: dans une lettre ouverte au président du gouvernement Felipe González, il avisait d'une part des dangers d'une « contamination de l'espagnol dans les communautés bilingues», et d'autre part de «la nécessité de protection de l'espagnol dans ces régions ${ }^{9}{ }^{9}$.

Dans le même ordre d'idées, quatorze ans plus tard (en 2008), une vingtaine d'intellectuels rédigent le "Manifeste en faveur d'une langue commune» assorti d'une «demande d'éclaircissement de la Constitution ${ }^{10} »$.

En même temps que les catégories Identité-différence se transforment, le lien entre identité et diversité se resserre, identité nationale équivalent à diversité linguistique, et identité nationale équivalent à identités historiques. Le tollé provoqué par ce manifeste entendait redonner au binôme Identité (nationale) - unité (linguistique) la place qui lui correspondait. Or, il reste un troisième élément dont on ne sait que faire: identités historiques, le grand exclu de la controverse.

8. «2. En materia de enseñanza y en lo que concierne a territorios distintos a sobre los cuales las lenguas regionales o minoritarias son tradicionalmente prácticas, las Partes se comprometen a autorizar, a estimular o a establecer, si el número de parlantes de una lengua regional o minoritaria lo justifica, una enseñanza en o de la lengua regional o minoritaria en las etapas apropiadas de la enseñanza. » Voir http://www.consello.org/ pdf/cartaeuropea92.pdf.

9. La nouvelle fut largement diffusée dans la presse nationale, « Retahila de reacciones », El País, 1/12/1994.

10. «Manifiesto por una lengua común», voir www.europapress.es, 17/06/08. Pour consulter les réactions à ce manifeste, voir http://www.elmundo.es/elmundo/2008/06/22/ espana/1214165304.html. 
Dans cet environnement conceptuel, on remarquera l'absence des notions telles que «minorité linguistique », «langue minoritaire » ou « langue propre ». Les appellations «langue minoritaire» et «langue minorisée » figurent dans la Charte européenne, pourtant approuvée par l'Espagne dans les années 1990 et ratifiée en 2001.

Deuxième construction: si la «connaissance» et «l'usage » de l'espagnol en tant que langue officielle est exprimée en termes de « droits et devoirs », les « modalités linguistiques » sont associées au « respect» et à la « protection », en une sorte de dédommagement moral qui n'exclut ni n'inclut leur pratique.

Troisième construction, la notion de « langues » au pluriel, assimilée à « modalités linguistiques » apparaît dans un entourage textuel particulier: la « promotion de la culture et de la recherche » (art. 148), et la « promotion $\mathrm{du}$ tourisme et du patrimoine monumental ». Ces langues seraient avant tout une option culturelle, plus qu'une obligation sociale (le castillan doit être maîtrisé par « tous les Espagnols »).

Le cadre constitutionnel restait suffisamment ouvert pour que, dans cette nébuleuse, chaque communauté historique réinterprète les notions initiales dans leurs propres statuts.

On peut, malgré tout, distinguer trois composantes communes dans les préambules respectifs des textes de ces trois communautés:

- Langue propre

- Normalisation

- Identité

Article 6 du Statut d'autonomie d'Euskadi : « L'euskera, langue propre du Peuple basque aura, comme le castillan, un caractère de langue officielle à Euskadi, et tous ses habitants ont le droit de connaître et d'employer les deux langues.

3. Nul ne saura être discriminé en raison de sa langue.

5. Euskera... patrimoine d'autres territoires basques. »

Les statuts premiers de l'euskera furent restreints par la Cour constitutionnelle dès lors que l'usage de la langue en termes de droits mais aussi de devoirs entrait en collision avec un texte commun dans lequel cette obligation n'était pas prescrite.

La Catalogne a modifié à son tour ses statuts en 2006 faisant valoir les devoirs envers le catalan, une rubrique refusée par la Cour constitutionnelle.

Or, l'une de ces trois notions communes fera l'objet d'interprétations 
divergentes: la normalisation. Elle va être comprise, soit comme «la mise au même niveau de deux langues ${ }^{11}$ ", soit comme " un processus à travers lequel la langue récupère progressivement ses fonctions formelles perdues et dans le même temps opère sur des secteurs sociaux, au sein de son propre territoire, où il n'y avait pas de locuteurs avant ». C'est ce deuxième positionnement qui sera adopté par le Congreso de la Cultura Catalana entre 1975 et 1977.

Une telle vision des choses peut être digne d'intérêt en ce qu'elle se distancie de l'objectif bilinguiste et rapproche la langue d'une autre notion: ce qu'Edward Said a conçu à la fin des années 1990 comme " affiliation ", c'est-à-dire, la possibilité de récupérer un passé pour composer un présent, bien que dans un processus divergeant de la filiation naturelle, ici grâce à une extension géographique et sociale possible ${ }^{12}$.

Si nous observons le dernier statut catalan (2006), l'état d'égalité ne serait pas une fin en soi mais une phase transitoire vers le «plein usage» du catalan.

Les préambules des lois de normalisation abordent donc des aspects divers:

Pour le catalan, la langue s'associe à une identité et est définie comme « un outil d'intégration communicative ». Les conséquences d'une perte de vitesse dans son usage seraient catastrophiques.

Dans les statuts galiciens sont évoquées « les conséquences désastreuses de deux cents ans de centralisme », ainsi que « l'appauvrissement croissant de la région » et de « l'usage du galicien en tant que facteur de cohésion à l'intérieur comme à l'extérieur des frontières de la région ».

Le texte légal basque répond au titre: « Loi de normalisation de l'usage de l'euskera. »C'est donc l'usage qui se normalise, non pas la langue, comme dans les deux autres cas. Les binômes langue-identité et identitédifférence sont exprimés dans le préambule en ces termes: «l'euskera est la marque la plus visible et objective de l'identité de notre peuple ».

Ce texte est promulgué sous la forme de Loi de base en 1982 mais, un an avant, le gouvernement basque avait déjà mis la main à la pâte en instaurant l'Institut pour l'euskaldunisation et l'alphabétisation des adultes (HABE), dépendant du Département régional de la culture.

La politique linguistique est ici greffée à la politique culturelle et

11. Rafael NinYoles, Idioma y poder social, Madrid, Tecnos, 1972, p. 75.

12. Pour un rappel du parcours vital et intellectuel de Said, voir Edward SAID, « Entre dos mundos », Fractal, n 9, avril-juin, 1998, 3e année, volume III, p. 93-112, traduction Jorge Brush. Original publié dans la London Revue of Books, n 7, mai 1998. 
les dotations budgétaires, allouées en grande partie à la publication de méthodes pédagogiques, en dépendent. L'HABE se dote également d'un cabinet de glottodidactique pour la recherche et la diffusion des méthodes d'enseignement de l'euskera. Aujourd'hui, il existe aussi un programme BOGA pour l'euskaldunisation via internet ${ }^{13}$.

Venons-en à la politique du livre comme l'un des segments où se concrétisent ces perspectives. Si nous évoquons ces exégèses et les initiatives qui en découlent, c'est que la politique du livre est étroitement liée aux programmes de reuskaldunisation. Entre 1990 et 2007, la production de livres en euskera aura triplé. Si les organismes publics ne vendent qu'un cinquième des livres qu'ils éditent ( $22 \%$ du total), les maisons privées facturent les deux tiers.

Quant au catalan, le statut approuvé en 2006 par la Communauté autonome de Catalogne le déclare " langue d'usage normal et préférentiel dans les administrations publiques ", et les citoyens catalans ont des droits et des devoirs sur elle. La totalité du statut est sous-tendue par un principe de liberté et d'égalité des citoyens dans le «développement de leur identité ${ }^{14} \gg$.

En Catalogne, la situation de diglossie ${ }^{15}$ affecte majoritairement la population immigrée et ses descendants, victimes d'un sentiment social d'infériorisation et faisant preuve d'un sous-usage du catalan au sein des communautés.

L'article 21 de la dernière loi de normalisation inscrit des «actions pour le développement» et une « discrimination positive », le 23 parle de « mesures positives d'action». Elles se traduisent en aides et subventions aux médias et en sanctions administratives en cas d'infraction de la loi ( offense civile»), des sanctions pour faute « légère » ou « grave ». En 2011, furent appliquées 226 amandes linguistiques, ce qui a rapporté 126000 euros à la Generalitat.

La Catalogne alloue un budget annuel de presque 32 millions d'euros à sa politique linguistique (budget de la direction générale de la politique linguistique pour 2011) et un total de 159 millions d'euros reversés aux différents secteurs publics (santé, travail, administration...).

Quant à la diffusion éditoriale du catalan, elle est stable et couvre autour

13. La Sociedad Cooperativa Urrats Euskaltegia fut fondée en 1988 par un groupe de professeurs qui comptaient une longue expérience dans la didactique de l'euskera.

14. Consultable sur http://www.gencat.cat

15. Pour une critique de cette notion, voir Lambert-Felix PrUDENT, «Diglossie et interlecte », Langages, ${ }^{\circ}$ 61, vol. 15, 1981, p. 13-38. 
de $10 \%$ de la production espagnole. Il faut dire que c'est la Communauté autonome qui édite le plus après Madrid, toutes langues confondues.

$77,5 \%$ des titres en catalan proviennent des maisons d'éditions asso-

En las publicaciones para niños se observaron las mayores diferencias, representando el $5,3 \%$ del total de las publicaciones en castellano, el 18,0\% del catalán y valenciano, el $16,6 \%$ del gallego y el $27,6 \%$ del euskera.

Los títulos en idiomas extranjeros representaron el 4,9\% de los títulos editados. Destacaron los libros en inglés $(67,3 \%$ del total), francés $(14,4 \%)$ y portugués $(7,0 \%)$.

Los títulos traducidos supusieron el $14,1 \%$ de la producción. Casi la mitad de ellos $(49,6 \%)$ fueron traducidos del inglés.

\section{Producción editorial por idioma de publicación}

Respecto a la proporción de títulos en las distintas lenguas nacionales, el castellano representó el $78,6 \%$ del total de títulos, el catalán y valenciano el $9,7 \%$, el gallego el $1,9 \%$ y el euskera el $1,2 \%$.

Títulos por idioma de publicación. Año 2012

\begin{tabular}{|c|c|c|c|c|c|c|}
\hline & Total & Castellano & $\begin{array}{c}\text { Catalán y } \\
\text { valenciano }\end{array}$ & Gallego & Euskera & Otros idiomas \\
\hline Titulos & 69.668 & 54.784 & 6.747 & 1.322 & 837 & $\overline{5.978}$ \\
\hline Porcentaje & 100 & 78,6 & 9,7 & 1,9 & 1,2 & 8,6 \\
\hline
\end{tabular}

ciées au Gremi d'Editors de Catalunya. $74 \%$ des titres en langue basque appartiennent aux maisons regroupées dans la Euskadiko Editoreen Elkartea. 70,6 \% des titres en galicien, de l'Asociación Galega de Editores.

Les interventions directes des gouvernements régionaux vont pour la plupart vers le secteur de l'éducation et de la formation. Les sommes allouées à la politique linguistique ont augmenté jusqu'en 2011. Seul pour le Plan général de promotion de l'usage de l'euskera, le Pays Basque a investi autour de deux millions d'euros (chiffres de 2011).

Les nouvelles normatives régionales diffèrent quant à l'utilité des publications scientifiques dans le projet de développement de la langue. Ainsi, en Catalogne, en 2010, l'Université de Barcelone publie un « Plan de langues » où elle réitère l'engagement avec la société catalane de protéger l'usage et la promotion de la langue propre de la Catalogne, conformément à ses statuts et au Règlement des usages linguistiques. Ce sont les deux textes de référence en vigueur.

Comment se concrétise cet engagement? En 2012, la Generalitat a financé 180 thèses rédigées en catalan (80000 euros), avec une présence homogène dans toutes les aires de la connaissance.

Dans le domaine des publications, les aides à l'édition et à la dif- 
fusion de livres de texte, manuels universitaires et livres scientifiques ou techniques sur support papier ou électronique en catalan ou traduits en catalan s'élèvent à 180000 euros (données de 2011). La Generalitat soutient aussi un programme de documentation scientifique promu par la Direction générale de la recherche, le département d'Économie de la connaissance (DEC) et l'Agence de gestion des aides universitaires et de la recherche (AGAUR). Ce programme a pour objet, entre autres, d'analyser l'impact du catalan dans la diffusion scientifique à l'international.

Les universités catalanes comptent aussi sur des aides pour la promotion et l'usage des langues dans le cadre universitaire (programme Interlingua 2012) pour garantir la présence et l'usage du catalan dans l'enseignement supérieur, en raison « de son caractère de langue propre et par responsabilité sociale ». Ces aides vont aussi vers l'anglais. En 2012, 726400 euros ont été destinés à l'organisation de cours de catalan pour les enseignants et enseignants-chercheurs (PDI), à l'élaboration d'épreuves universitaires pour l'accréditation de connaissances linguistiques, à la conception de ressources virtuelles (test, outils d'évaluation, etc.).

Pour l'euskera, plusieurs critères sont appliqués dans le but de favoriser l'usage de cette langue dans l'enseignement: la thèse en langue basque des enseignants-chercheurs titulaires aura valeur de thèse internationale si elle atteint la plus haute mention. Si l'enseignement se fait en euskera, la rétribution courante est valorisée de $20 \%$ (décret 64 du 29 mars 2011).

Sur le plan de la recherche à proprement parler, il n'existe pas de mesures effectives pour favoriser l'usage de l'euskera. C'est le volontarisme des équipes et des laboratoires qui compte. Le gouvernement offre cependant 20 bourses annuelles de quatre ans pour la préparation d'une thèse en euskera. Il y a aussi un prix bisannuel organisé par l'Académie de la langue basque pour les meilleures thèses rédigées en cette langue (un premier prix et deux accessits).

Pour la Galice, le budget alloué à la promotion de la langue est en franc déclin : 21,5 millions d'euros en 2009, 15,6 en 2010, 9,3 en 2011 et 8,6 en 2012.

Si nous contrastons à présent les initiatives et les résultats, la Galice est le territoire dont la situation s'avère la plus alarmante du point de vue linguistique. L'usage du galicien est en perte de vitesse dans les zones urbaines, le décalage entre la connaissance et l'usage se creuse de plus en plus (à peine la moitié des locuteurs l'utilisent habituellement). Seul $20 \%$ des enfants de maternelle reçoivent l'enseignement en galicien. En 2008, dix ans après le premier projet de normalisation, la Galice avait déjà 
perdu la moitié de ses locuteurs natifs. En parallèle, le nombre de locuteurs n'utilisant pas la langue régionale aura doublé.

Pour limiter la débâcle, un décret de dynamisation du galicien en dehors du circuit universitaire est publié en 2010. Son but, « renforcer la dimension communicative du galicien, faciliter à l'élève une offre d'enseignement qui l'aide à percevoir l'utilité de la langue et le rende capable de l'appliquer correctement ». Ce décret serait « respectueux de la situation sociolinguistique de chaque établissement $\gg$. Les initiatives seraient centrées sur les activités péri et extrascolaires (équipe de dynamisation) ${ }^{16}$.

Si les faits signalés au début de cette présentation prouvent sans doute la difficulté à s'orienter dans un espace symbolique propre, à assumer de nouveaux espaces selon la perception de tout un chacun, les données montrent les degrés d'efficacité de la mise en œuvre des politiques linguistiques à partir de ces différences de perception.

L'imbrication du « fait culturel », de la position idéologique, spirituelle ou politique des individus et des collectifs au sein d'une communauté reconnue légalement comme telle a créé chez nous des réactions de défense et de contre-défense de ce que l'on considère comme " propre " ou « commun », et met à mal régulièrement la triade Langue-Peuple-État.

Les tensions et les liens entre la politique et les langues vont, non pas vers la langue même, considérée comme une "altérité » mais vers le bilinguisme, c'est-à-dire, vers une unité abstraite duelle dont les résolutions sont asymétriques. Abstraite et malléable, car le bilinguisme dans nos régions est à interpréter comme une préférence pour l'usage du castillan (cf. communiqués et plaintes des différentes Tables pour la libre expression). Les contre-arguments bilinguistes appellent à une "réalité » sociolinguistique et réfutent la valeur «langue propre» au profit de la « faculté langagière ».

Au-delà des querelles régulières, exprimées sous forme de manifestes, pyrotechnies médiatiques, signatures d'élites légitimant des pratiques politiques sensées ou ouvertement suicidaires, les transformations sont intervenues avec peu de sensibilité à l'égard des langues en situation subalterne, qui ont connu des réactions allant de l'ultra-défense à l'autocensure. Mais aussi avec peu d'empathie des pouvoirs envers une partie de la population, qui se voyait contrainte à l'apprentissage et à la

16. En Catalogne: $37 \%$ de la population considère le catalan comme une langue d'identification contre $46 \%$ pour le castillan et $8,8 \%$ se dit bilingue. http://www.idescat.cat/ territ/BasicTerr?TC $=6 \& \mathrm{~V} 0=3 \& \mathrm{~V} 1=3 \& \mathrm{MN}=1 \& \mathrm{~V} 3=1047$ 
communication dans l'une ou l'autre langue, obligée d'accepter des canons auxquels elle ne se sentait pas ou plus identifiée.

D'où découle la nécessité affichée par certaines communautés de tracer des territoires d'interface où ses langues seraient valorisées, en dépassant la revendication d'un héritage usurpé, d'une filiation, sous peine de se voir reléguées au muséisme, et encourageant de nouvelles représentations capables de déclencher de nouveaux processus d'affiliation.

Des initiatives sont tout de même menées, comme le prouve l'exemple de ces livres captifs. Seraient-ils un signe d'autocensure, d'autorépudiation? Ces langues en cohabitation fragile retrouveront-elles un jour leurs pleines fonctions sociales?

\section{Bibliographie et sitographie}

ESCARPIT Robert et Ronald BARKER, La faim de lire, Paris, Presses Universitaires de France, 1973. El deseo de leer, Barcelona, ed. Península, eds. De Bolsillo, col. Ensayo, 1974 (trad. Luis A. Gil López).

HofFMANN Charlotte, «Monolingualism and Bilingualism, Cultural Pluralism and National Identity: Twenty Years of Language Planning of Contemporary Spain», dans Sue Wright (dir.), Monolinguism and Bilinguism. Lessons from Canada and Spain, Clevedon-Philadelphia, Multilingual Matters, 1996.

KREMNITZ Georg, « Du "bilinguisme" au "conflit linguistique", cheminement de termes et de concepts », Langages, ${ }^{\circ}$ 61, vol. 15, 1981, p. 63-74.

NEVADO Felipe Aparicio, "Langues "régionales" versus castillan: une guéguerre interculturelle hispano-ibérique », dans Karin Dietrich Chénel et Marc Weisser (dir.), L'interculturel dans tous ses états, Paris, Orizons, coll. «Universités », 2012, p. 109-121.

NINYOLES Rafael, Idioma y poder social, Madrid, Tecnos, 1972.

PARRY R. Gwynedd, «Bilingual legal scholarship in Wales », dans X. Arzoz (dir.), Bilingual higher education in the legal context. Group rights, state policies and globalisation, Leiden-Boston, Martinus Nijhoff Pub, 2012.

PRUDENT Lambert-Felix, « Diglossie et interlect», Langages, $\mathrm{n}^{\circ}$ 61, vol. 15, 1981, p. 13-38.

SAID Edward, «Entre dos mundos », Fractal, n 9, avril-juin 1998, année 3, volume III, p. 93-112 (trad. Jorge Brush). 
http://www.culturagalega.org, 17/01/08.

http://www.economiaefacenda.org

http://www.elpais.com, 1/12/1994.

http://www.europapress.es, 17/06/08.

http://www.gencat.cat/generalitat/cat/estatut/index.htm

http://www.ine.es/jaxi/menu.do?type=pcaxis \&path=/t12/p401\&file=inebase

http://www.la-moncloa.es/constitucion.es.pdfPe nobit enim qui tempore ipienti cone volores et idus etur, am aligniet atis comnis si odit quasintibus que vel et facest lab ide plab ilignam usciaessi omnihit voluptatur rectorest as audandunt et omnia sapienimil estio eosti quiam laccusam nem. Con repudaecae ilit faccust, te nis anis exceressi adis consendi re nestio que iminctur, to dignitas sum laut andandi genimus inulles tibust, unt.

Isque sequam, volupta quam que quiderit remperi orupta quae perrum dolut event re, et qui dolorunt pa in rehendandi inum ut vel iliam, voluptas molumquation repro bla platur, aut ipsam que quid ut que cus sit eos esenihil ipsandi uta volupta doluptatet quo minimint quassum qui odigendi tem ut ad et ut eaturitas atium ex et fuga. $\mathrm{Mi}$, consequo core et ut ernatur a sequo offic tempedigenim voluptini ipitibusdam, consequod mos ad quist voluptas aute voluptione culparum sant, verum fugitaquid quo cusam as rae. Ut a ditatiassit dolorenis estotat ibusae nist, optius, qui ut volenditium cor adit quibust iberuptatas maxim laborro vidunti nonsed mil ipsam nonse nisit, si autaqua temquunt idunt illuptatio. Etus rati verisquatur? Ipsaeptur aut et ligeniatem sit, aut laborrum ipsandiore maionec estion es venihillanto oditati ncius.

Siti ullitatum quid qui res modio quam, consequ amusande nit, veliquiat laborem ossitaspid quo quamenihilia cullent peditate ipsae nestiost que molessera volorae ra seceperumqui officia sitibusdae maion re laut demque molum, ipsam que pliat.

Num simende bissequis net lautemp orerspi enetus ut laut dis dolent es rerspel endebis dolupta tinisciet ex eatem facepudae sequae. Axim repedis ex et andicius ex experum harum am quiatetur, se simet ex es nobitia nimusan delibearum ilibus moles dolorep eriscia simus asit eumquunt.

Optatiunt pro blautes cipicipsam latem endi doloreicil il ea es eos rati accabor erorum voluptia parum sim estiisita sam voluptis minum ilitat aut qui utam qui blaut facestiore vellaborum fugia aut qui simi, quas a voluptatem quam, suntiae prescimus as distis conseque sendanda des que estiant exceped ut ut iusam a quatquas dolesti corit mos aut quas untibusa eum fugiandia que lacerest fugit rem explaces eatem volo et ut offic to qui rehento rehenia spiendi tateceaqui doluptatem restis doloreptur, qui ute et et et eicae doloraecto occum ut hit vellab ipid quae. Nemporem nobit officiam, sit, cus sitatio tenduntium soluptatet voluptation comnis quis soluptaquis rent ut volupta tquasperem et quas sae porit volupit, 